\section{Estereotipos negativos de la vejez en estudiantes de establecimientos rurales}

\section{Negative stereotypes of old age in students of rural establishments} establishments

\author{
Juana Cortés Recabal ${ }^{1}$ \\ José León Pino ${ }^{2}$ \\ Jocelyne Chandia Bustos ${ }^{3}$ \\ Carla Muñoz Gómez ${ }^{3}$ \\ Abigail Orellana Navarrete ${ }^{3}$ \\ Giovanna Robbiano Muñoz
}

1. Enfermera. Magíster en Familia. Departamento de Enfermería. Universidad del Bío-Bío. Chillán. Chile.

2. Enfermero. Magíster en Ciencias Biológicas. Escuela de Enfermería. Facultad de Salud. Universidad Santo Tomás. Chile.

3. Enfermera. Licenciada en Enfermería. Universidad del Bío-Bío. Chillán. Chile.

*Autor para correspondencia.

Correo electrónico: jcortes@ubiobio.cl (Juana Cortés Recabal).

Recibido el 24 de mayo de 2018; aceptado el 16 de octubre de 2018

\section{RESUMEN}

Objetivo: Determinar el nivel de negatividad de los estereotipos de la vejez en estudiantes de establecimientos rurales, Región de Nuble, Chile. Metodología: Estudio descriptivo de corte transversal sobre una muestra de 114 estudiantes de $5 .^{\circ}$ a $8 .^{\circ}$ básico de dos establecimientos rurales en Chile. La información fue recolectada mediante la aplicación del cuestionario de estereotipos negativos de la vejez (CENVE). Resultados: El 53,5\% ( $\mathrm{n}=61$ ) de los estudiantes obtuvo un alto nivel de estereotipo negativo, donde la dimensión más negativa fue salud en todas las variables estudiadas. Conclusión: Los estudiantes percibieron un alto nivel de estereotipo negativo hacia la vejez, por lo que resulta necesario realizar intervenciones que se enfoquen en cambiar la visión negativa que poseen respecto a este grupo etario y, de esta manera, lograr que los ancianos se sientan integrados y valorados en la sociedad actual.

PALABRAS CLAVE: Estudiantes, estereotipo, anciano, envejecimiento.

\section{ABSTRACT}

Objective: to determine the level of negativity of stereotypes of old age in students of rural establishments, Nuble Region, Chile. Methodology: descriptive cross-sectional study on a sample of 114 students from 5 th to 8 th grade of two rural establishments, in Chile. The information was collected through the application of the questionnaire of negative stereotypes of old age (CENVE). Results: $53.5 \%(n=61)$ of the students obtained a high level of negative stereotype, where the most negative dimension was health in all the variables studied. Conclusion: the students perceived a high level of negative stereotype towards old age, therefore, it is necessary to carry out interventions that focus on changing the negative view they have regarding this age group; and in this way, make the elderly feel integrated and valued in today's society.

KEYWORDS: Students, stereotypes, aged, aging.

\section{- INTRODUCCIÓN}

Los estereotipos están ligados al surgimiento de prejuicios y discriminación ${ }^{1}$ y se encuentran dentro de las llamadas representaciones sociales, las cuales corresponden a influencias que las personas reciben de la sociedad y los orientan dentro de un contexto social ${ }^{2}$. De este modo, identifican una idea predeterminada por la sociedad hacia un grupo de la comunidad, el cual presenta un conjunto de rasgos típicos que los caracteriza ${ }^{1}$. En la sociedad actual, los adolescentes perciben la vejez como una etapa de aislamiento del trabajo, con predisposición a enfermedades y pérdida de fuerza para los enfrentamientos diarios ${ }^{2}$.

Por otra parte, los niños en etapa escolar comienzan a abrir su mundo a nuevas actividades y pensamientos, lo cual los hace más susceptibles a adoptar las ideas de la sociedad, más aún los niños con menos autoestima, los cuales son más influenciables en el intento por incluirse en ella ${ }^{3}$. Es así como en la vida escolar uno de los factores influyentes en cuanto a los estereotipos negativos de la vejez son los textos escolares ${ }^{4}$.

Desde la visión de enfermería, los estereotipos de la vejez y los numerosos prejuicios existentes en la sociedad pueden condicionar los cui- dados que se otorguen a los ancianos, a consecuencia de las actitudes sociales adheridas, que van desde el rechazo a la sobreprotección o infantilización de aquellos. Ante esto, se hace necesario profundizar en esta área mediante la investigación, para poder otorgar cuidados adecuados a los ancianos, que se enfoquen en promover la autonomía y participación activa en el proceso salud y enfermedad. Y el profesional de enfermería es quien debe saber contener situaciones en las que predominen dificultades, ya sean personales, familiares y/o sociales en el entorno del anciano que pudieran llevarle a presentar complicaciones 5 .

Sumado a lo anterior, existe exigua evidencia sobre este tema en Chile, aún más en el área rural. A nivel internacional, España y Costa Rica presentan investigaciones que abordan la problemática ${ }^{6,7}$. Pero es el sector urbano donde se pueden identificar más investigaciones tanto nacionales como internacionales ${ }^{1,8-10}$.

Considerando lo anteriormente expuesto, el propósito de esta investigación es determinar el nivel de negatividad de los estereotipos de la vejez en estudiantes de establecimientos rurales de la Región de Núble en Chile, y de este modo generar evidencia que sea un primer paso en estudios que incorporen esta temática en el país. 


\section{- OBJETIVO}

Determinar el nivel de negatividad de los estereotipos de la vejez en estudiantes de establecimientos rurales, Región de Nuble, Chile.

\section{- MATERIAL Y MÉTODO}

Estudio descriptivo transversal en estudiantes de $50^{\circ}$ a $80^{\circ}$ básico de establecimientos rurales, Región de Nuble, Chile. La población total fue de 172 estudiantes de dos establecimientos, de los cuales se excluyó a aquellos que no fueron capaces de comprender las instrucciones para contestar el cuestionario y/o cursaban por segunda o más veces el mismo nivel. Se obtuvo una muestra total de 114 estudiantes que aceptaron participar previa firma del consentimiento informado por representante legal.

Los datos fueron recogidos mediante dos instrumentos: Cuestionario de caracterización sociodemográfica de la población, que fue elaborado por los autores de este estudio, y que consta de 7 preguntas, donde las cuatro primeras determinan las características sociodemográficas de los estudiantes y las siguientes buscan conocer la relación de este con el adulto mayor, mediante preguntas de respuestas dicotómicas cerradas. El segundo instrumento correspondió al Cuestionario de estereotipos negativos de la vejez (CENVE) ${ }^{11}$, conformado por 15 ítems en total, con formato de respuesta graduada tipo Likert de cuatro valores. Su medición se basa en tres factores: salud, motivacional-social y carácter-personalidad, con 5 ítems cada uno. El factor salud evalúa lo relacionado con el deterioro de la salud, cognitivo, de la memoria y enfermedades mentales; el factor motivacional-social lo componen los ítems relacionados con la pérdida de interés por las cosas, incapacidad para resolver problemas e interés disminuido por el sexo; y, finalmente, el factor carácter-personalidad destaca por indicar que los defectos de las personas se agudizan con la edad. La puntuación del cuestionario va de 15 a 60 puntos, y la puntuación por factor fluctúa entre 5 y 20 puntos. La clasificación de la puntuación del cuestionario es la siguiente: $a$ ) de 15 a 28 puntos corresponde a muy bajo nivel de estereotipo negativo, b) de 29 a 39 puntos corresponde a bajo nivel de estereotipo negativo, c) de 40 a 50 puntos corresponde a alto nivel de estereotipo negativo, d) de 51 a 60 corresponde a muy alto nivel de estereotipo negativo hacia los adultos mayores. Los cuestionarios fueron administrados por dos encuestadores por curso, una vez que los estudiantes recibieron las instrucciones para su cumplimentación. Dado el carácter autoadministrado de los cuestionarios, cada estudiante contestó de manera individual en su puesto de trabajo en la clase. Se buscó favorecer un ambiente propicio en la sala para la aplicación objetiva y uniforme de los cuestionarios, con buena iluminación, libre de ruidos y distractores. Adicionalmente, se contó con la presencia de un docente para resguardar el orden dentro del aula. Se obtuvieron las autorizaciones del comité de ética y de los establecimientos educacionales participantes.

Para el procesamiento de datos se utilizó el software SPSS Statistics v.15, donde se analizaron variables cualitativas mediante frecuencias observadas como valores absolutos y frecuencias relativas porcentuales, y las variables cuantitativas por medio de media y desviación típica.

\section{- RESULTADOS}

Las edades de los estudiantes se encontraban entre los 9 y los 13 años, un $57,9 \%(\mathrm{n}=66)$ fueron mujeres, un $71,9 \%(\mathrm{n}=82)$ pertenecía a la religión católica, un 22,8\% $(\mathrm{n}=25)$ a la evangélica y un 5,3\% (n = 6) refirió ser de otra confesión (testigos de Jehová, Judaica, mormón y ateo). La evaluación del nivel de estereotipo negativo por medio del CENVE arrojo que el 53,5\% ( $\mathrm{n}=61)$ de los estudiantes obtuvo un alto nivel de estereotipo negativo (tabla 1 ). Con respecto a la media de la puntuación obtenida en los tres factores, el 14,86 fue en el factor salud, el 13,56 en el factor motivacional-social y el 12,81 en el factor carácter personalidad (tabla 2). En el momento de describir las puntuaciones de los factores respecto al sexo de los estudiantes, se obtuvo una diferencia de 1,52 puntos en el factor salud, 0,75 puntos en el factor motivacional-social y 0,27 puntos en el factor carácter personalidad (tabla 3). Cuando se evalúan las puntuaciones de los factores respecto a la religión, se observa que aquellos de la categoría "otras" presentaron una puntuación media superior en los factores salud y motivacional-social con respecto a los que pertenecían a la religión católica y a la evangélica (tabla 4). Respecto a la relación que presentaban los estudiantes con los ancianos y la media de puntuaciones obtenidas en los diferentes factores, se observó que no hubo grandes diferencias en las medias obtenidas en los tres factores entre los estudiantes que tenían a sus abuelos vivos, vivían o no con los ancianos o compartían con los ancianos (tabla 5).

Tabla 1. Distribución de los estudiantes participantes en el estudio según nivel de estereotipo

\begin{tabular}{|l|c|c|}
\hline Estereotipo & N & $(\%)$ \\
\hline Muy bajo estereotipo negativo & 7 & 6,14 \\
\hline Bajo nivel de estereotipo negativo & 35 & 30,70 \\
\hline Alto nivel de estereotipo negativo & 61 & 53,51 \\
\hline Muy alto nivel de estereotipo negativo & 11 & 9,65 \\
\hline Total & 114 & 100,00 \\
\hline
\end{tabular}

Tabla 2. Medidas de resumen de la puntuación obtenida en las tres dimensiones que evalúa el nivel de negatividad del estereotipo vejez de los estudiantes participantes del estudio

\begin{tabular}{|l|c|c|c|c|c|}
\hline Dimensiones & Amplitud teórica & $\overline{\mathbf{x}} \pm \mathbf{D E}$ & $\mathbf{P}_{\mathbf{2 5}}$ & $\mathbf{P}_{\mathbf{5 0}}$ & $\mathbf{P}_{\mathbf{7 5}}$ \\
\hline Salud & $5-20$ & $14,86 \pm 3,00$ & 14,30 & 15,00 & 15,42 \\
\hline Motivación social & $5-20$ & $13,56 \pm 2,93$ & 13,02 & 14,00 & 14,10 \\
\hline Carácter personalidad & $5-20$ & $12,81 \pm 3,39$ & 12,18 & 13,00 & 13,44 \\
\hline
\end{tabular}

a Corresponde a la puntuación mínima y máxima por cada dimensión del instrumento.

$\bar{x} \pm D E$ : media aritmética \pm desviación estándar; $\mathrm{P}_{25}$ : percentil 25; $\mathrm{P}_{25}$ : mediana; $\mathrm{P}_{75}$ : percentil 75 
Tabla 3. Puntuación de las tres dimensiones, que evalúa el nivel de negatividad del estereotipo vejez de los estudiantes participantes del estudio desagregado por sexo

\begin{tabular}{|l|c|c|c|}
\multirow{2}{*}{ Sexo } & \multicolumn{3}{|c|}{ Nivel de negatividad del estereotipo vejez } \\
\cline { 2 - 4 } & Salud & Motivación -social & Carácter-personalidad \\
\hline Femenino & $15,50 \pm 2,81$ & $13,88 \pm 2,79$ & $12,92 \pm 3,48$ \\
\hline Masculino & $13,98 \pm 3,07$ & $13,13 \pm 3,08$ & $12,65 \pm 3,30$ \\
\hline
\end{tabular}

Media aritmética \pm desviación estándar

Tabla 5. Puntuación de las tres dimensiones, que evalúa el nivel de negatividad del estereotipo vejez de los estudiantes participantes del estudio, desagregado por relación que sostiene con el anciano

\begin{tabular}{|l|c|c|c|c|}
\hline \multirow{2}{*}{ Relación con el anciano } & \multicolumn{3}{|c|}{ Nivel de negatividad del estereotipo vejez } \\
\cline { 2 - 4 } & & Salud & Motivación -social & Carácter-personalidad \\
\hline \multirow{2}{*}{ Abuelo vivo } & Sí & $14,85 \pm 3,02$ & $13,55 \pm 2,91$ & $12,84 \pm 3,40$ \\
\hline \multirow{2}{*}{ Vive con anciano } & No & $14,92 \pm 3,00$ & $13,67 \pm 3,23$ & $12,50 \pm 3,53$ \\
\hline Comparte con anciano & Sí & $14,27 \pm 3,27$ & $13,40 \pm 3,31$ & $13,21 \pm 3,87$ \\
\hline & No & $15,16 \pm 2,86$ & $13,50 \pm 2,75$ & $12,47 \pm 3,14$ \\
\hline
\end{tabular}

Al menos 30 minutos tres veces a la semana.

Media aritmética \pm desviación estándar.

\section{- DISCUSIÓN}

Este estudio identifica los niveles de estereotipos negativos en estudiantes de establecimientos rurales, donde destaca el predominio de un alto nivel de estereotipo negativo, seguido de un bajo nivel de estereotipo negativo; por tanto, estos resultados nos sugieren que en Chile, de acuerdo con la evidencia científica, indistintamente en el área urbana o rural existe una percepción negativa hacia la vejez por parte de la población ${ }^{12,13}$. Por otro lado, estos resultados difieren de dos investigaciones, una realizada en Colombia y otra en España, donde predominó un bajo nivel de estereotipo negativo $^{14,15}$. Estas diferencias podrían deberse a los diferentes grupos de estudios analizados en estas investigaciones, donde se estaría comparando escolares de 9 a 13 años con adultos, además de las vivencias propias de cada persona, donde la cultura cumple un papel importante. En esta misma línea, un estudio de estereotipos, de tipo cualitativo realizado en España a una comunidad educativa, arrojo que los estudiantes asociaban la vejez con decadencia, enfermedad, pérdida, deterioro e incapacidad ${ }^{10}$, características que se asemejan a las encontradas en el presente estudio, donde predomina un alto porcentaje de estereotipo negativo, asociado a características del anciano como incapacidad, deterioro mental y dependencia, entre otros. Estos resultados se muestran preocupantes en el momento de analizar un estudio realizado a adolescentes de un instituto rural donde el 93\% piensa que los ancianos son una carga para la sociedad y un $68 \%$ refiere que son menos tolerables ${ }^{6}$, lo cual estaría indicando que la visión que se tenga de los ancianos desde la etapa escolar se conservaría en la adolescencia y quizás en la adultez. Debido a la limitación del estudio por ser descriptivo y no permitir establecer relaciones entre variables para determinar causalidad, se buscó otra explicación a esta problemática, tomando como referente las visiones de las corrientes más clásicas del pensamiento del ser humano, donde filósofos
Tabla 4. Puntuación de las tres dimensiones que evalúa el nivel de negatividad del estereotipo vejez de los estudiantes participantes del estudio desagregado por religión

\begin{tabular}{|l|c|c|c|}
\multirow{2}{*}{ Religión } & \multicolumn{3}{|c|}{ Dimensiones de la escala CENVE } \\
\cline { 2 - 4 } & Salud & Motivación -social & Carácter-personalidad \\
\hline Católica & $14,65 \pm 2,92$ & $13,20 \pm 2,52$ & $12,60 \pm 3,29$ \\
\hline Evangélica & $15,27 \pm 3,11$ & $14,27 \pm 3,67$ & $13,50 \pm 3,57$ \\
\hline Otras $^{\text {a }}$ & $16,00 \pm 3,85$ & $15,50 \pm 3,73$ & $12,67 \pm 4,23$ \\
\hline
\end{tabular}

a Testigos de Jehová, Judaica, mormón y ateo.

Media aritmética £ desviación estándar 


\section{- LIMITACIONES}

Dentro de las limitaciones se encuentran:

1. La investigación es de naturaleza descriptiva; por tanto, no permite determinar relaciones o causalidad del fenómeno.

2. Presencia de alumnos inscritos en los establecimientos rurales, que no concuerda con la población real que asistía a las clases. Por este motivo, se procedió a cambiar el total de población con la que real- mente asistía como alumno regular; por tanto, los no inscritos fueron excluidos de la población de estudio.

3. El espacio físico utilizado para la recolección de datos no contó en ocasiones con el silencio requerido, debido a la infraestructura de las aulas

\section{Conflicto de intereses}

Los autores declaran no tener ningún conflicto de intereses.

\section{- BIBLIOGRAFÍA}

1. Franco M, Villarreal E, Vargas E. Estereotipos negativos de la ve ez en personal de salud de un Hospital de la Ciudad de Querétaro México. Revista médica Chile [Revista en internet] 2010 [acceso e 23 de septiembre de 2015];138:988-93. Disponible en: http://www. scielo.cl/scielo.php?pid=S0034-98872010000800007\&script=sci_arttext

2. Álvaro JL. Representaciones Sociales. Diccionario Crítico de Ciencias Sociales. Theoria [Revista en internet] 2010 [acceso 23 de septiembre de 2015]. Disponible en: http://pendientedemigracion.ucm es/info/eurotheo/diccionario/R/representaciones sociales.htm

3. Guerrero A. Desarrollo del niño durante la etapa escolar. Manual de Pediatría [Monografía en internet]. Escuela.med.puc.cl; 2015 [acceso 8 de octubre de 2015]. Disponible en: https://issuu.com/vpenela/ docs/manual-pediatria-puc---pediatria-ambulatoria/39

4. Ramírez T. El texto escolar: una línea de investigación en educación. [Revista en internet]. Ve.scielo.org. 2003 [acceso 8 octubre 2015]. Disponible en: http://ve.scielo.org/scielo.php?script=sci_arttext\&pid=S0798-97922003000200003

5. Sánchez I, Castañeda H. Prevalencia de depresión en adultos mayores de 65 años y propuesta de intervención preventiva. Geroinfo. Publicación de Gerontología y Geriatría [Revista en internet]; 2008 [acceso 8 de octubre de 2015];3(3). Disponible en: http://www.sld $\mathrm{cu} /$ galerias/pdf/sitios/gericuba/articulo_depresion.pdf

6. Lirio J. Fernández M. La pedagogía social en la universidad, Investigación formación y compromiso social [Monografía en internet]. Naullibres. Valencia. 2012 [acceso 8 de octubre de 2015]. Disponible en: https://books.google.cl/ books? id=3B9Y9LXJfTAC\&pg=PT60\& $\mid p g=P T 60 \& d q=$ percep cion+de+escolares+rurales+sobre+vejez\&source=bl\&ots=a dcC5a 5tn\&sig=SSY3GR60RATiSNROfovSLPBqqgc\&hl=es\& $\mathrm{sa}=X \& v e d=0 \mathrm{CFg} 06 \mathrm{AEwCGoVChMl6bLi8r6syAIV0060Ch3IZ}$ OvU\#v $=0$ nepage $\& q=$ percepcion $\% 20$ de $\% 20$ escolares $\% 20$ rurales $\% 20$ sobre $\% 20$ vejez\&f=false

7. Granados M. Investigadora examina la percepción de los niños sobre los adultos mayores. Congreso de Gerontología [Revista en internet] 2014 [acceso el 8 de octubre de 2015]. Disponible en: http:// www.ucr.ac.cr/noticias/2014/10/08/investigadora-examina-la-perepcion-de-los-ninos-sobre-los-adultos-mayores.htm

8. Jorquera P. Vejez y envejecimiento: Imaginarios sociales presentes en los textos escolares oficiales del Ministerio de Educación chileno. [Revista en internet]. HYPERLINK "http://revistamad.uchile. cl/"Revistamad.uchile.cl. 2010 [acceso 27 de septiembre de 2015] Disponible en: https://revistamad.uchile.cl/index.php/RMAD/article/view/13642/13920

9. De Freitas MC, Ferreira MA. Vejez y persona anciana: representaciones sociales de adolescentes escolares. Revista Latino Americana Enfermagem [Revista en internet] 2013 [acceso 23 de septiembre de 2015];21:2-7. Disponible en: http://www.scielo.br/pdf/rlae/ v21n3/es 0104-1169-rlae-21-03-0750.pd

10. Cerquera A, Alvarez J, Saavedra A. Identificación de estereotipos y prejuicios hacia la vejez presentes en una comunidad educativa de Florida Blanca. Psychologia Avances de la disciplina [Revista en internet] 2010 [acceso 8 de octubre de 2015];4(11):73-87. Disponible en: http://redalyc.org/articulo.oa?id=297224086007
11. Sánchez Palacios C. Estereotipos negativos hacia la vejez y su relación con variables sociodemográficas, psicosociales y psicológicas. [Tesis doctoral]. Málaga: Universidad de Málaga; 2004.

12. Contreras Llanos M, Fuentes Badilla L, Rivas Pérez A Solís Carrasco M. Percepción de los estudiantes de enseñanza media sobre estereotipos de la vejez en liceos municipalizados de una comuna de Chillán, 2014 [tesis de pregrado]. Chillán: Universidad del Bío Bío; 2014

13. Fierro V, Hernández N, Pantoja A. Estereotipos de la vejez percibidos en escolares de $5^{\circ}$ y $6^{\circ}$ año del colegio Darío salas de la comuna de Chillán Viejo [tesis de pregrado]. Chillán: Universidad del Biobío: 2015

14. Aristizábal M, Morales A, Salas B, Torres Á. Estereotipos negativos hacia los adultos mayores en estudiantes universitarios. Cuadernos Hispanoamericanos de Psicología [Revista en internet] 2009 [acceso 14 de noviembre de 2015];9 (1):35-44. Disponible en https://issuu. com/universidadelbosque/docs/vol9

15. Larraz Gómez B, Martín Peña J. Imagen social de la vejez: un acercamiento a los principales estereotipos y las repercusiones discriminatorias en las personas mayores [tesis doctoral]. Zaragoza: Universidad de Zaragoza; 2013

16. Fernández Ballesteros R. La psicología de la vejez. Universidad Autónoma de Madrid, España, 2002. [acceso 05 de junio de 2016]. Disponible en: http://www.encuentros-multidisciplinares.org/Revistan\%C2\%BA16/Roc\%C3\%ADo\%20Fernandez\%20 Ballesteros.pdf 